\title{
What is Open Angle Glaucoma?
}

\section{IAN GRIERSON}

\section{London}

The question "What is Open Angle Glaucoma?" has been asked by research workers and clinicians alike but, despite surgical and medical advances, the development of more effective means of assessing the glaucomatous eye and a multitude of experimental studies we are still far from having a reasonable answer. The pathological mechanisms which bring about cupping of the optic nerve head and progressive field loss are as mysterious and controversial as the mechanisms by which blockage of the outflow pathways and chronic elevation of intraocular pressure (IOP) develop. However the "question", for the purposes of the present study is restricted to pathological developments in the outflow pathways.

Many proposals have been put forward concerning the pathological events which lead to dysfunction of the outflow tissues in primary open angle glaucoma (POAG) (Table 1). To a large extent, the pathological changes which may (or may not) distinguish open angle glaucoma are also features of the ageing outflow system..$^{1-5}$ Therefore the idea that glaucomatous pathology may be one of degree rather than kind is a reasonable one, worthy of detailed consideration.

Progressive decrease in the cell population is now a well documented process in the ageing trabecular meshwork. ${ }^{1-3}$ What is not so clear is whether there is an excessive loss of cells associated with glaucomatous pathology. Some morphological evidence would strongly support such a concept, ${ }^{2}$ other data is less convincing ${ }^{3}$ and, for that matter, one group of research workers have emphasised focal hyperplasia as a feature of glaucoma rather than pronounced cell loss. ${ }^{6}$
Depletion of the cell population (with excessive reparative fibrosis of the survivors) may be fundamental to the early pathology in glaucoma. If so, open angle glaucoma would have obvious similarities to the pathological events which take place in the corneal endothelium and which results in corneal decompensation. The object of the present study therefore, was to determine whether we could find evidence of disproportionate cell loss in the glaucomatous meshwork, attempt to explore mechanisms which could produce loss of meshwork cells and to see whether such a loss could represent part of the answer to our question "What is Open Angle Glaucoma?"

Over the past 5 years, we have conducted morphological investigations on the outflow pathways of normal tissue and several hundred trabeculectomy specimens. Some of this material in conjunction with findings from tissue culture studies form the basis of the present report.

\section{Materials and Methods}

All specimens were processed for light microscopy, transmission electron microscopy (TEM) and scanning electron microscopy (SEM) using conventional techniques. TEM was conducted in either a JEOL 100 c or an Hitachi 600 , whereas SEM specimens were viewed in an Hitachi S520.

\section{Qualitative morphology}

A total of 44 human eyes provided the material for studies of normal trabecular meshwork. The tissue had previously been used for a morphological investigation of age changes in Schlemm's canal. Essentially the group was a mixture of specimens removed in treatment of posterior segment tumours and post-mortem eyes. The age range was from birth to 92 years $\left(\right.$ see $\left.^{7}\right)$. 
Table I Features associated with ageing, primary open angle glaucoma (POAG) and secondary open angle glaucoma (SOAG).

\begin{tabular}{lccc}
\hline Feature & Aging & $P O A G$ & SOAG \\
\hline Narrowing of collector channels & no & yes & $?$ \\
Closure of Schlemm's canal & no & yes & yes \\
Loss of giant vacuoles & yes & yes & yes \\
Accumulation of extracellular materials in the endothelial meshwork & yes & yes & yes \\
Thickening of trabeculae & yes & yes & yes \\
Accumulation of wide spacing collagen & yes & yes & yes \\
Fusion of trabeculae & yes & yes & yes \\
Increased phagocytosis & yes & yes & yes \\
Thickened scleral spur & yes & yes & yes \\
Hyperplasia at anterior border of the meshwork & yes & yes & yes \\
Cellular activation & $?$ & yes & yes \\
Loss of meshwork cells & yes & yes & yes \\
\hline
\end{tabular}

As part of an on-going clinicopathological survey, in excess of 700 trabeculectomy specimens have been sent to our laboratory for morphological investigation. Included with each specimen was a data sheet which provided details such as IOP on presentation at the glaucoma clinic, IOP immediately before surgery, patient's age, sex, type of glaucoma, history of antiglaucoma medication, facility (if available) etc. The specimens came from various centres throughout the country. For most, surgery had been performed following the failure of maximal medical therapy. With others surgery was performed after the failure of, at least, one antiglaucoma medication. However specimens also were supplied from a centre where trabeculectomy was conducted as the first line of treatment. These patients went to surgery soon after their first presentation at clinic and had either no or minimal medication. Minimal medication being either 2 per cent pilocarpine or 0.5 per cent timolol for a period of less than two weeks prior to surgery. Therefore we had an extremely valuable group, which could on the whole be considered to be at a relatively earlier stage of glaucomatous development, for comparison with, on the one hand the more established POAGs with a history of medication and on the other hand, our normal specimens of a comparable age range.

The series included approximately 50 secondary open angle glaucomas (SOAG) of which 7 were specimens of pigmentary glaucoma. Comment on the morphological features of trabeculectomies from patients with pigmentary glaucoma will form part of this report.

\section{Quantitation}

For effective quantitation only the largest trabeculectomy specimens which contained, in meridional section, Descemet's membrane and corneal endothelium at the anterior limit and the scleral spur at their posterior limit were deemed suitable (Fig. 1). Thus the light microscopic quantitative study was restricted to 30 trabeculectomy specimens of 'early' POAG (i.e. surgery was the first form of antiglaucoma treatment) and 27 specimens of 'later' POAG. For the trabeculectomies, a total of 3-4 semi-thin meridional sections were cut with a $10 \mu \mathrm{m}$ spacing between each section. The sections were stained with toluidine blue and examined under an oil immersion $\times 100$ objective lens of the light microscope (Vickers). An eyepiece frame was placed beneath the mid-portion of Schlemm's canal so that its upper limit lay on the endothelium lining the trabecular aspect of the canal but excluded the uveal meshes closest to the chamber angle. Counts were made of the total number of meshwork cell nuclei within or touching the limits of the frame and these were subdivided into nuclei from cells in the endothelial meshwork adjacent to Schlemm's canal and nuclei in the remaining, more extensive, trabecular portion. All cytoplasmic profiles containing nuclei and within the frame were classified as being either pigmented or nonpigmented.

The normals were randomly selected from those in our control series which were 40 years and over. For the 18 control normal specimens, one tissue block was selected at random per eye and then subjected to the same light microscopic evaluation as that conducted on the trabeculectomies. In addition to test the validity of the frame count as being representative of the meshwork as a whole, frame counts were conducted on a series of 30 normals of all ages. The frame count data was then correlated against nuclear counts from the whole meshwork 


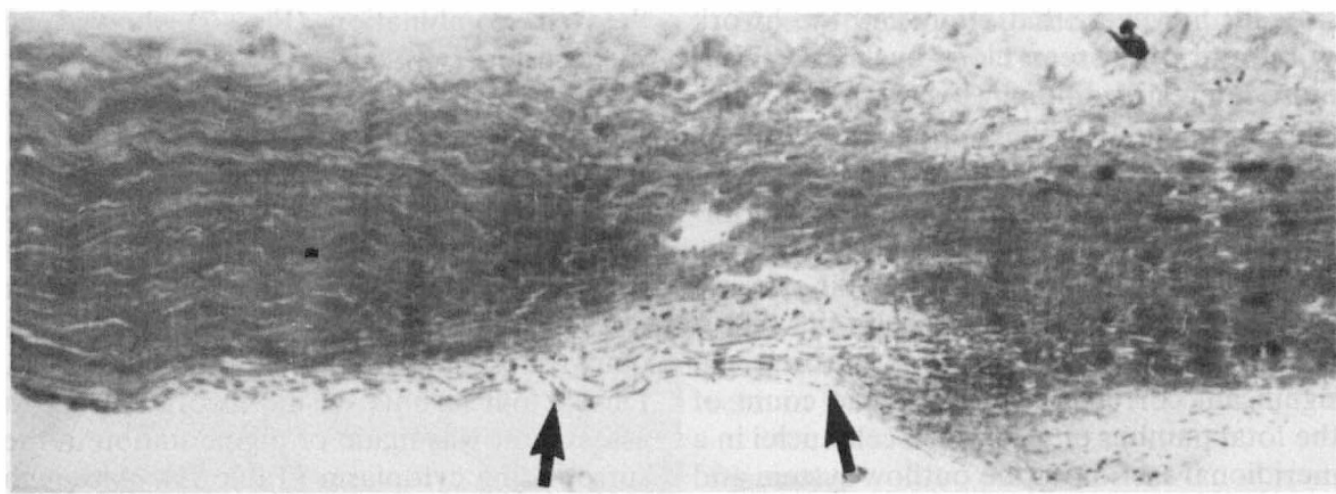

Fig. 1. Light micrograph showing trabeculectomy specimen. The trabecular meshwork is indicated (arrows) $(\times 45)$.

(as seen in one meridional section) for the appropriate subject.

\section{Tissue culture}

To study some effects of phagocytosis on meshwork cells cultures of bovine meshwork at third passage ${ }^{8}$ were fed materials such as latex microspheres $(0.1$ and $0.8 \mu \mathrm{m}$ diameter), carmine, yeast and bovine iris melanin granules for various time periods up to 1 week and at concentrations varying from 50 to 50,000 particles per cell. The cells were monitored by phase microscopy (Olympus), TEM and SEM.

\section{Results}

\section{Qualitative observations in POAG}

Approximately 80 per cent of our trabeculectomy specimens had trabecular tissue present but much was of indifferent quality either being damaged at the time of removal or containing only a modest segment of meshwork. However in such a large series sufficient specimens were of the necessary quality to provide useful information. On qualitative examination of Schlemm's canal by TEM, the canal endothelium was generally thinner in the trabeculectomy specimens than the age matched normals. Discontinuities between adjacent endothelial cells were common in the trabeculectomy specimens on both aspects of the canal but these were rare in the normals. It would be unwise to make too much of this because of the possibility that the 'gaps' in the endothelium may be the consequence of surgical trauma. Total closure of Schlemm's canal and replacement by fibrous tissue and debris was noted in some of our 'early' POAG specimens but was a more common finding in those who had had maximal medical therapy prior to surgery (Fig. 2). Accumulation of extracellular material in the endothelial meshwork of the drainage wall of Schlemm's canal, trabecular thickening and trabecular fusions were pronounced in many of the POAG trabeculectomies but the variation between and within the specimens was impressive (Figs. 2 and 3). Given that all three features are known to increase in the ageing eye,$^{3-5}$ only detailed quantitative evaluation by TEM could provide meaningful data and this was not done in the present study.

Twenty 'late' POAG specimens, processed for SEM, were orientated so that the trabecular mesh adjacent to the chamber angle could be examined with ease. In particular, the most anterior portion of the mesh was of interest where the trabeculae merged with the peripheral cornea. Overgrowth of the anterior mesh by flattened plate-like cells beyond that seen in appropriate normal controls was noted in 18/20 (Fig. 4). Only 2 'early' POAGs were examined in a similar manner and neither of these exhibited cellular overgrowth. Specimens of both 'early' and 'late' POAG, orientated so that the deeper trabeculae could be examined, frequently had areas of cellular denudation (Fig. 5A). Denuded trabeculae were a feature of the ageing normal tissue, but they seemed to be more easily found and identified in the POAG specimens. Again the possibility of surgical trauma must be con- 
sidered; however, that migratory meshwork cells were often seen close by, made this a more interesting finding (Fig. 5B).

\section{Quantitative light microscopy of meshwork nuclei in POAG}

For our group of 30 normals of all ages we were gratified to find that despite the difference in sample size, there was a strong and significant correlation between the count of the total number of meshwork cell nuclei in a meridional section of the outflow system and the corresponding frame count from the central portion of the meshwork $(r=0.9584$, $\mathrm{p}<0.001)$. Thus within obvious limits what occurred to the cell population in the frame was a reasonable reflection of what was happening to the whole population.

The group of 18 control normals (all over 40 years), 30 early POAGs and 27 'late' POAGs were of comparable age (no significant difference Student T-test) (Table II). The 'early' POAGs had a lower mean number of nuclei in the frame counts than the normals but the difference was not significant. On the other hand, the count for 'late' POAGs was significantly lower than the normals $(\mathrm{p}<0.001$ Student T-test) (Fig. 6). If the count was subdivided into trabecular and endothelial meshwork interesting trends were evident. The trabecular count in both 'early' and 'late' specimens was significantly lower than the controls $(\mathrm{p}<0.001$ Student T-test). However, although the count of endothelial meshwork nuclei was similar in the normals and the 'late' group, it was significantly higher in the 'early' POAGs than the other two groups $(\mathrm{p}<0.01$ Student T-test) (Fig. 6). Given the trend for progressive decrease in counts of total nuclei and trabecular meshwork nuclei from normal tissue to 'late' POAG (Fig. 6) and the percentage of nuclei in the endothelial meshwork of both 'early' and 'late' POAGs ( 34 per cent and 36 per cent) was twice that in the normal series (20 per cent).

The mean IOP of the 'early' POAGs at the time of surgery was significantly lower than that of the 'late' POAGs $(\mathrm{p}<0.05$; Student T-test) (Table II). Attempts to correlate IOP at the time of surgery with numbers of nuclei in 'early' POAG, 'late' POAG or 'early' and 'late' in combination (Fig. 7) showed no relationship.

Phagocytosis by meshwork cells

During ageing there is a progressive increase in the incidence of meshwork cells containing melanin. ${ }^{3}$ It is thought that the melanin is released from the iris particularly in the older eye, circulates in the aqueous humour and is phagocytosed by the native meshwork cells. During our counts of meshwork nuclei an assessment was made of pigmentation in the surrounding cytoplasm (Table II). Although the nuclear count progressively decreased from normal tissue, to 'early' POAGs and, finally, to 'late' POAGs (Fig. 6) the numbers of profiles containing melanin increased. The percentage of melanin containing profiles in the normal control group was approximately 16 per cent but was over 40 per cent in the 'late' POAGs (Table II).

In culture, bovine meshwork cells were seen to phagocytose a wide range of biotic (IgG coated microspheres, melanin granules, yeast) and abiotic (uncoated microspheres, carmine dye) particles. TEM showed that the particles were incorporated into phagosomes and histochemical staining indicated an acid phosphatase positive reaction around the engulfed materials (Fig. 8A). When the cells were fed large amounts of particulate material on a daily basis, they incorporated more and more particles until they lysed and were lost from the culture plate. For example if the cells were fed 50,000 IgG-coated microspheres per cell daily, cell death was evident at 4 days and by 7 days few, if any, viable cells were left (Figs. 8B and C). For that matter, if bovine iris melanin granules were fed to the cells at as low a level as 1,000 per cell, cell death was even more marked, with minimal numbers on the culture plate at the 4 day period.

\section{Features of SOAGs}

If large amounts of particulate material such as haematogenous debris, erythroclasts, pseudoexfoliation material and melanin enter the outflow system in situ, a secondary open angle glaucoma can develop. As well as these particles causing a simple blockage, part of the pathology would also seem to be related to phagocytosis (Fig. 9). If the meshwork cells 


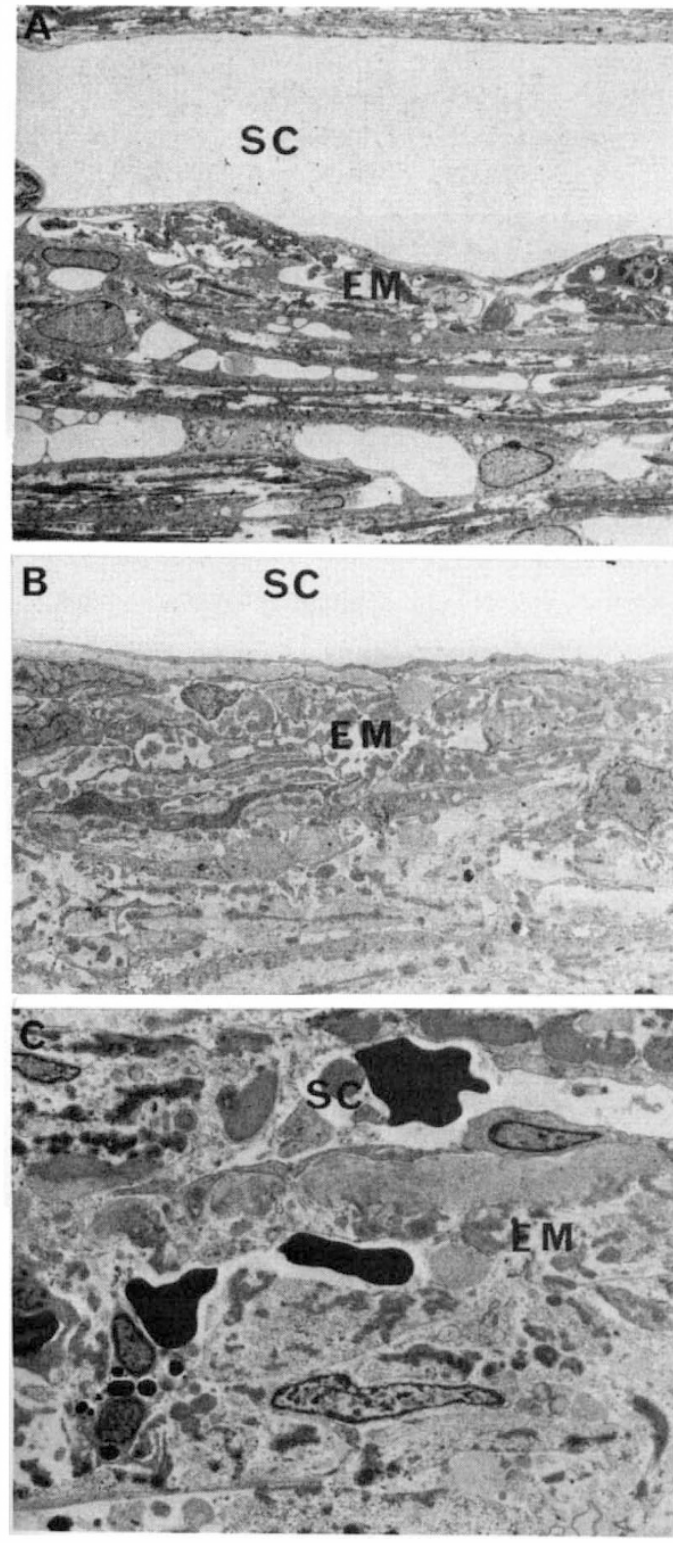

Fig. 2. TEMs of the outflow system in POAG showing the endothelial meshwork (EM) under Schlemm's canal (SC). A) "Early" POAG, B) "late" POAG and C) "late" $P O A G$ with disruption and partial closure of the canal $(A \times 2,000 ; B \times 3,000 ; C \times 5,500)$.

take up too much debris, they can become mobile phagocytes (Fig. 9B) and migrate from the meshwork. With materials like melanin, local cell lysis can also take place (Fig. 9C). The frame counts are of 3 of the seven cases of pigmentary glaucoma are shown in Table III.
Taking their age into account, the cell population was depleted. The meshwork exhibited pronounced reparative fibrosis, trabeculae were fused together and thickened, the endothelial meshwork was packed with extracellular material and in the most severely affected patient Schlemm's canal was partially replaced. Interestingly, SEM showed that plate-like flattened cells had extended over the most anterior portion of the meshwork as seen in POAG.

\section{Discussion}

The incidence of cell nuclei in the meshwork was significantly lower in POAG trabeculectomies from patients who had had a substantial regimen of antiglaucoma medication prior to surgery than in age matched controls. It seemed that in these 'late' glaucoma specimens, the decrease in nuclei (cells) was associated with the trabecular portion of the meshwork whereas the endothelial meshwork cell incidence was similar in normals and 'late' POAG. As such, the findings were similar to those of Alvarado et al. ${ }^{2}$ The technique of frame counting was adopted here so that areas of meshwork such as the meshes adjacent to the chamber angle and which are sometimes prone to surgical trauma could be avoided. Alvarado counted the meshwork population from a whole meridional section of the outflow tissue reconstructed from photomicroscopic montages. Of course, our frame count did correlate well with the total count for the series of normals but this need not hold for the trabeculectomies. A more important difference was that Alvarado's group ${ }^{2}$ took a measurement of the area occupied by intertrabecular spaces which could then be excluded. Therefore the incidence of cell nuclei was expressed per unit area of tissue as an assessment of cellularity. The area of intertrabecular space has not been taken into consideration in the present evaluations. Given that trabeculectomy will probably result in some compression of the intertrabecular spaces between removal and fixation due to loss of the pressure head, then the POAG counts relative to the normals will if anything be higher than they should be!

By far the most important group in the present investigation were the 'early' POAGs. 


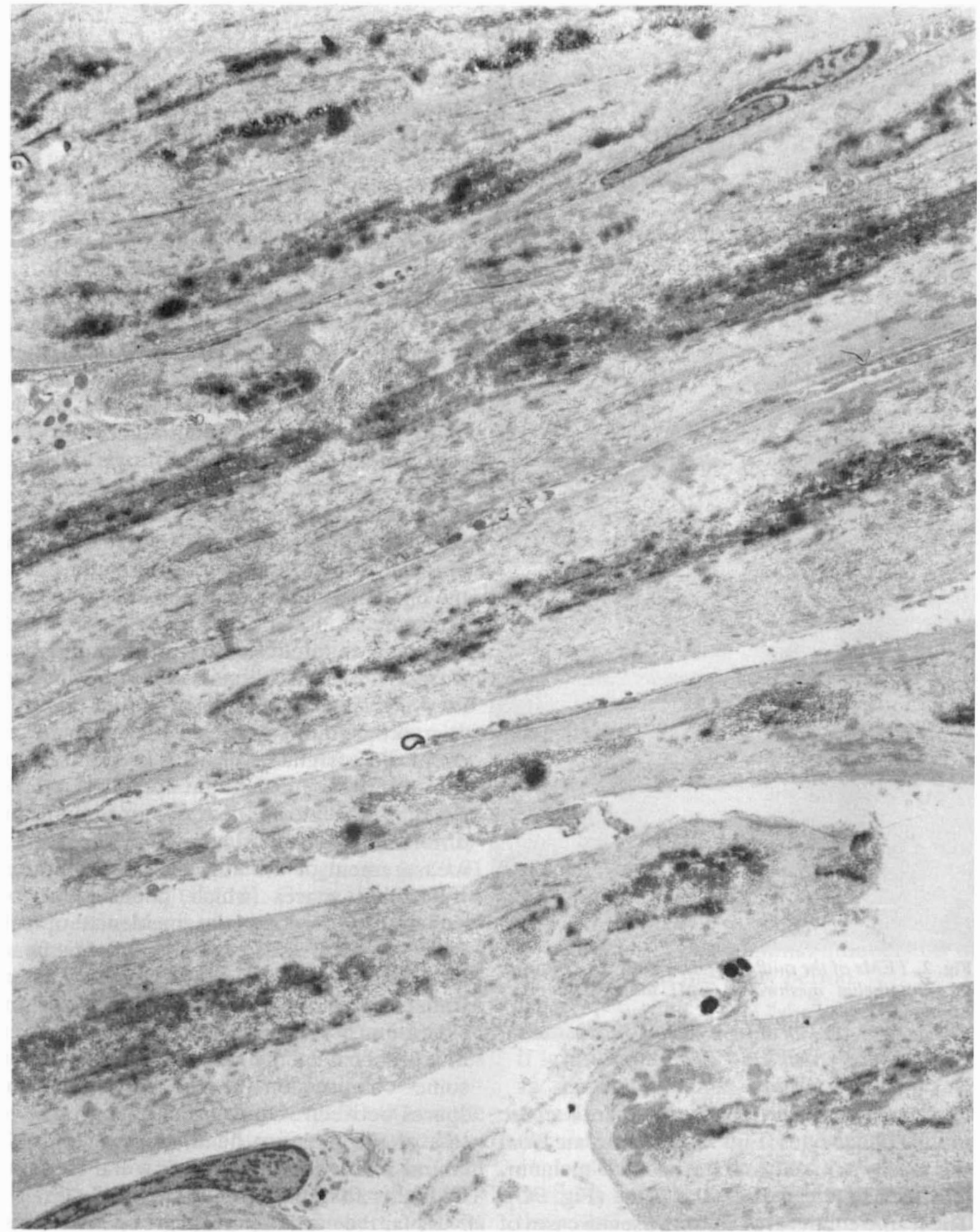

Fig. 3. TEM showing trabecular thickening and fusion in "late" $P O A G(\times 3,000)$. 


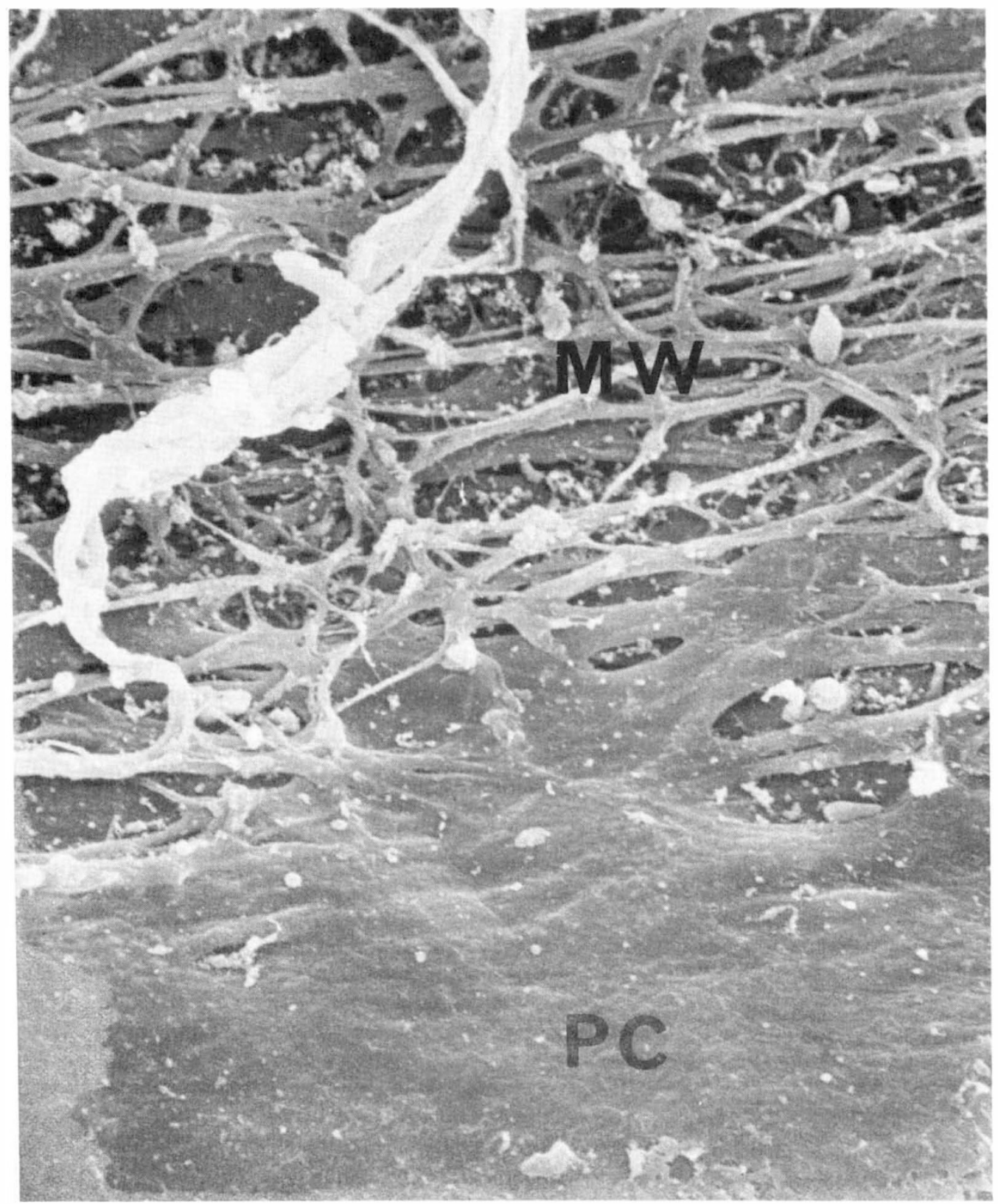

Fig. 4. SEM showing plate-like cells $(P C)$ which have extended over some of the anterior portion of the meshwork $(M W)$ in the "late" group $(\times 500)$. 

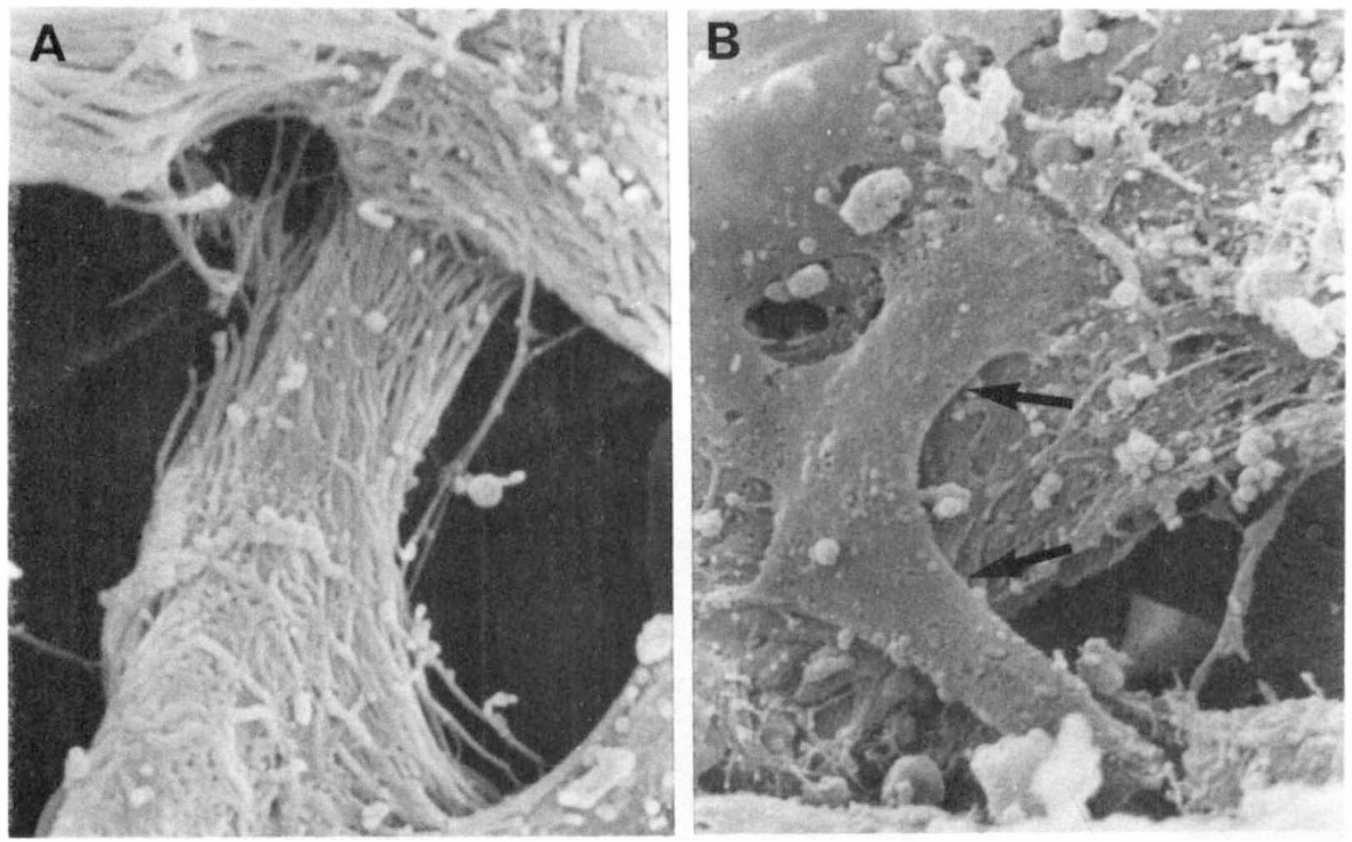

Fig. 5. SEM of trabeculae in A) "early" and B) "late" POAG. A) shows the collagen core of a denuded trabecula and $B)$ shows a migratory meshwork cell (arrows) $(A \times 6,000 ; B \times 10,000)$.

The classification of POAG specimens into 'early' and 'late' presumed that patients first presenting at the glaucoma clinic and then going to surgery as quickly as possible have had a shorter duration of glaucomatous pathology than the patients who have had antiglaucoma medication for an appreciable period prior to surgery. However, it could be predicted that the glaucomatous status of the patients at first presentation may have been quite variable; so that without such details as optic cupping and visual field loss, groupings of 'early' and 'late' are open to criticism. None the less the group as a whole, without or with minimal antiglaucoma medication, was material which provided valuable insights into the initial pathological events associated with POAG. Although there was pronounced variation between specimens, and some of the untreated POAGs exhibited advanced pathological change, the untreated or 'early' POAGs had less advanced pathology than the 'late' POAGs.

Interestingly there was not a significantly lower total nuclear count in the 'early' POAGs but when the count was subdivided the trabecular meshwork count in the 'early' POAGs was significantly lower than the

Table II The mean \pm standard deviation of age, IOP and per cent incidence of pigmented cell profiles in the specimens subjected to quantitative evaluation.

\begin{tabular}{|c|c|c|c|c|c|c|}
\hline Specimens & $\begin{array}{l}\text { Average age } \\
\text { (yrs) }\end{array}$ & $(T$-test) & $\begin{array}{l}\text { IOP at } \\
\text { Surgery }\end{array}$ & $(T$-test $)$ & $\begin{array}{l}\text { Pigmented } \\
\text { meshwork } \\
\text { cells }(\%)\end{array}$ & $(T$-test $)$ \\
\hline $\begin{array}{l}\text { Normals over } \\
40 \text { years of age }\end{array}$ & $66.9 \pm 14.0$ & (NS) & - & & $16.1 \pm 11.3$ & $(\mathrm{p}<0.05)$ \\
\hline $\begin{array}{l}\text { POAG No or minimal } \\
\text { previous medication }\end{array}$ & $70.3 \pm 8.3$ & (NS) & $24.7 \pm 5.5$ & $(\mathrm{p}<0.05)$ & $26.3 \pm 17.1$ & $(\mathrm{p}<0.01)$ \\
\hline $\begin{array}{l}\text { POAG with previous } \\
\text { medical therapy }\end{array}$ & $70.1 \pm 14.1$ & & $29.4 \pm 6.7$ & & $44.5 \pm 19.8$ & \\
\hline
\end{tabular}




\section{NUCLEI}

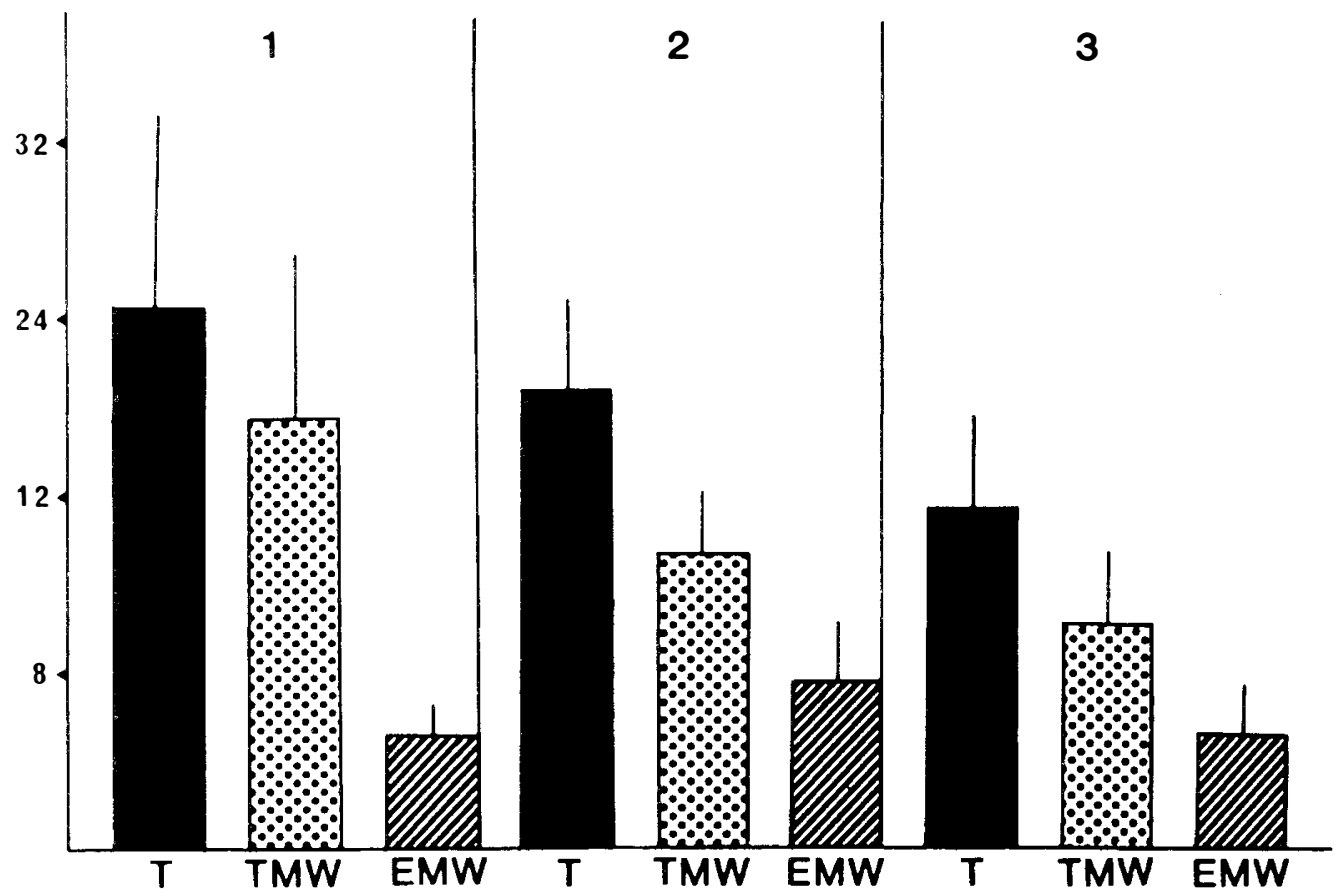

Fig. 6. Histogram of mean and standard deviation for total nuclei $(T)$, trabecular meshwork nuclei (TMW) and endothelial meshwork nuclei (EMW) in (1) normal controls over 40 years, (2) "early" POAG and (3) "late" POAG.

normals but the endothelial meshwork count was significantly higher! Given the difference between the trabecular meshwork and the endothelial meshwork it may be that our preliminary observation that decreased cellularity was important only in the advanced disease state $^{3}$ was incorrect.

Let us assume for the purposes of this discussion, that differential cell loss is a major factor in the early pathology of POAG then how is it brought about?

Although human meshwork cells can proliferate in culture ${ }^{9}$ their capacity for replication in situ appears to be so limited as to be negligible. So the number of meshwork cells we have at birth are all that we possess! The steady decline in cells throughout life has been thought of in terms of a wear-and-tear action on the delicate meshwork tissue,,$^{1-3}$ possibly a life time of variable aqueous flow and contraction of the ciliary muscle being major contributory factors. Low level trauma produced by muscle action or aqueous flow may evoke, in addition to cell death, cell migration and reparative fibrosis. Certainly more severe trauma, as produced by ciliary muscle disinsertion ${ }^{10}$ or laser trabeculoplasty, ${ }^{11}$ are associated with meshwork cell 'activation', migration and fibrosis.

In addition we have previously discussed the possibility that phagocytosis of materials in the aqueous humour (particularly melanin) could also contribute to the depletion of the meshwork cell population. ${ }^{3}$ Meshwork cells engaged in phagocytosis can lyse; being nonprofessional phagocytes they appear neither

Table III The IOP, number of nuclei per frame count ( \pm standard deviation) and per cent of pigmented cell profiles for 3 cases of pigmentary glaucoma.

\begin{tabular}{lcrc}
\hline Age & IOP at surgery & $\begin{array}{c}\text { Total nuclei } \\
\text { in frame }\end{array}$ & $\begin{array}{c}\text { Pigmented } \\
\text { cells in frame }\end{array}$ \\
\hline 34 & 30 & $17.0 \pm 3.7$ & $94 \%$ \\
50 & 35 & $14.0 \pm 2.2$ & $91 \%$ \\
46 & 26 & $5.7 \pm 1.5$ & $93 \%$ \\
\hline
\end{tabular}


Table IV Flow chart indicating possible interrelationships of pathological events as seen in ageing, POAG and SOAG.

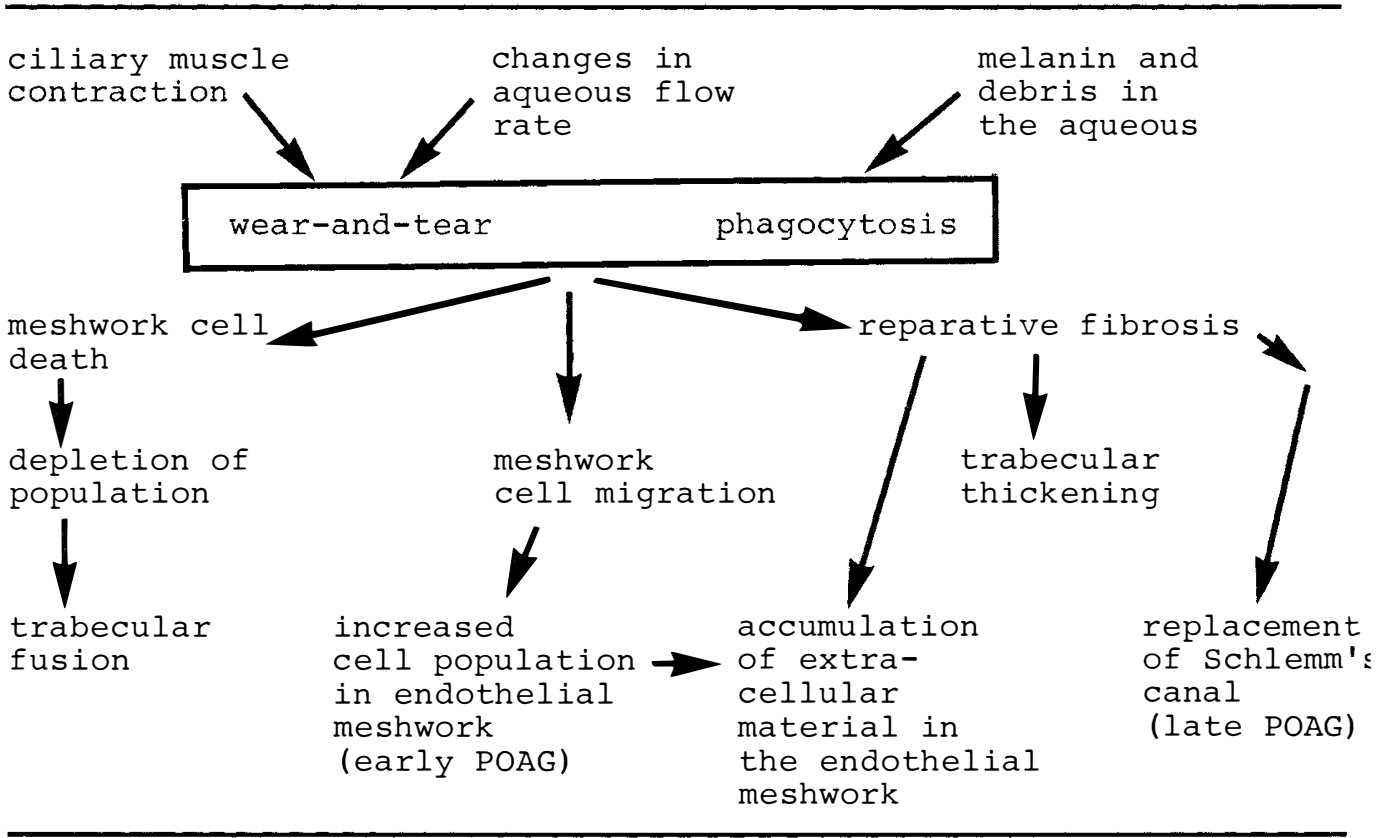

to have the equipment necessary to digest melanin nor, as shown by our tumour culture studies, do they have a mechanism to switch off from eating.

Even if the phagocytic meshwork cells do not die they can become mobile and migrate from the outflow system as has been described in experimental studies where debris was introduced into the outflow system. ${ }^{12}$ For that matter in the present study, pigmentation was more pronounced in POAG than in age matched normals and in pigmentary glaucoma. Pigmentary glaucoma was associated with hyperpigmentation: migration of meshwork cells from the trabeculae and meshwork cells lysis was commonplace. A markedly depressed cell population characterised the three specimens of pigmentary glaucoma analysed by frame count. A case is not made for phagocytosis as being the sole mechanism for depletion of the meshwork cell population in ageing, POAG and SOAGs (like pigmentary glaucoma) or even the major mechanism. It is merely a mechanism which probably contributes to cellular loss and is therefore worthy of investigation (Table IV).
A widely held proposal discussed by Rohen and Lutjen-Drecoll ${ }^{13}$ is that phagocytosis by meshwork cells is a helpful process "for cleaning in the intertrabecular spaces of debris and particles" and presumably preventing blockage of the narrowest aqueous drainage pathways. However because particles as large as red blood cells can negotiate the trabecular meshwork with ease, then a system of phagocytic self cleaning is unnecessary. I consider that meshwork cell phagocytosis, far from being an important function, is an embryolical relic which when evoked is harmful to the tissue rather than helpful.

It can be seen from Table I that the pathology associated with POAG is, for the most part, seen also in the SOAGs like pigmentary glaucoma. Indeed many of the alterations associated with glaucoma are progressively more evident in the ageing but normal outflow system. Thus in ageing, progressive cell depletion brought about by wear-and-tear or phagocytosis, leads to trabecular thickening, trabecular fusions and accumulation of extracellular materials in the endothelial meshwork. ${ }^{1-5}$ These, and other changes probably 


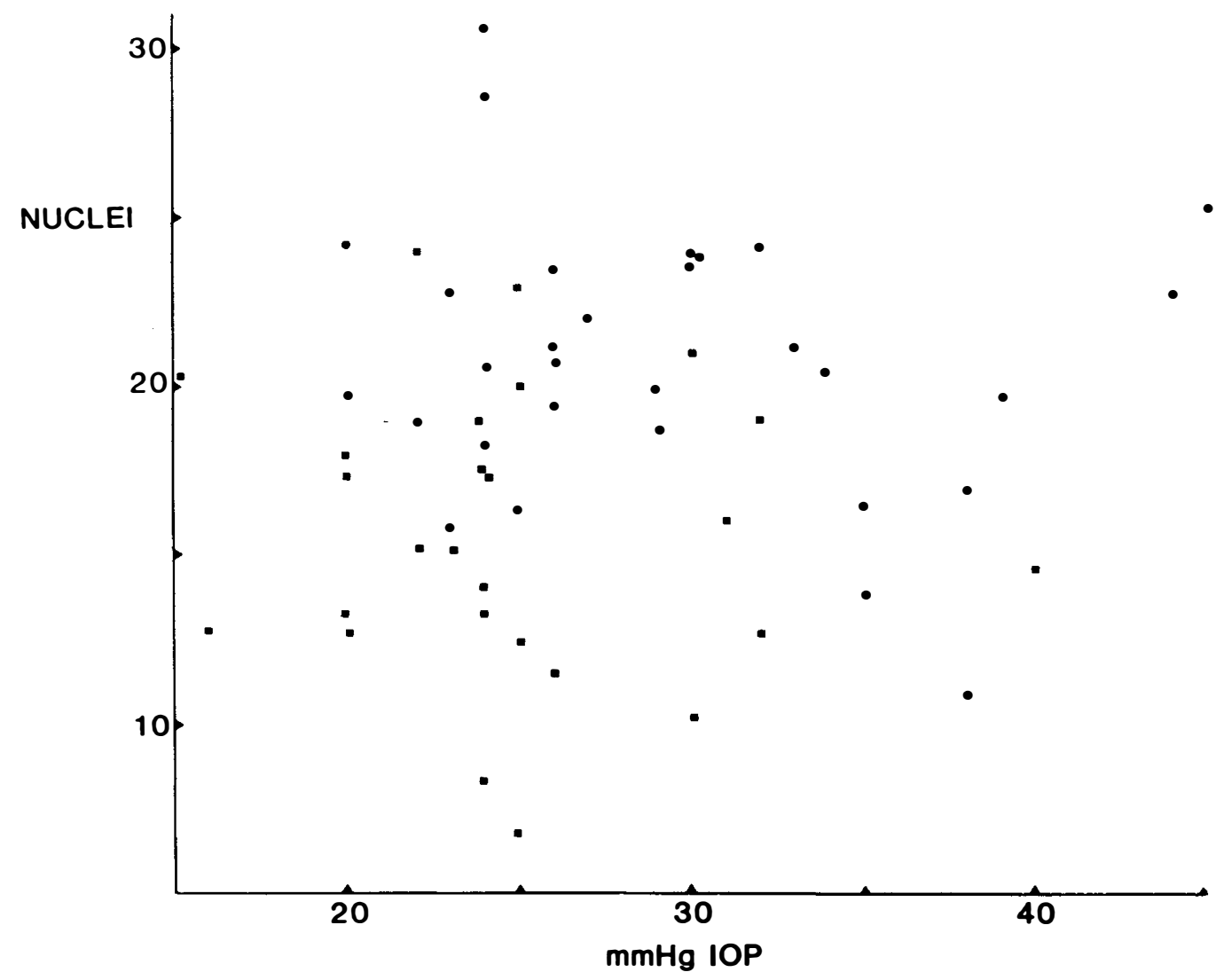

Fig. 7. Scattergram showing the lack of a significant relationship between total nuclei in frame count and IOP. Circles = "early" POAG and squares = "late" POAG.

result from a combination of cell loss and reparative activity evoked in the remaining meshwork cell population. Facility is likely to decrease throughout life but IOP in the normal ageing population remains fairly stable probably because there is a concomitant decrease in aqueous production to offset the facility change. ${ }^{14}$

However in POAG it may be a reasonable working hypothesis to assume that the meshwork cells are more prone to wear-and-tear and/or more at risk from the effects of phagocytosis. Thus in early POAG there were fewer meshwork cells on the trabeculae and the increase in the endothelial meshwork cell population could be explained by the migration of 'activated' trabecular cells into the region. With an 'activated' and enlarged cell population, accumulation of extracellular materials such as glycosaminoglycans, ${ }^{15}$ fibro- nectin $^{16}$ and elastic-like elements ${ }^{17.18}$ in the endothelial meshwork is predictable (Table III). In more advanced POAG, overall cell depletion was more clear cut and may account for the excessive trabecular fusion and thickening and extension of the scleral spur, as described by Fine et al. ${ }^{6}$ Increase in wide spacing collagen ${ }^{19}$ and basement membrane material ${ }^{16}$ within trabeculae coupled with the release of matrix vesicles ${ }^{20}$ and proliferation of peripheral corneal endothelium over trabecular meshes ${ }^{2}$ are indicative of metabolic and synthetic disturbances in the surviving meshwork cell population. Replacement of Schlemm's canal is a particularly advanced feature but entirely consistent with the overall trend (Table I).

Thus we come down to the proposition that the pathology of POAG in the outflow system is one of an exaggerated ageing process. 

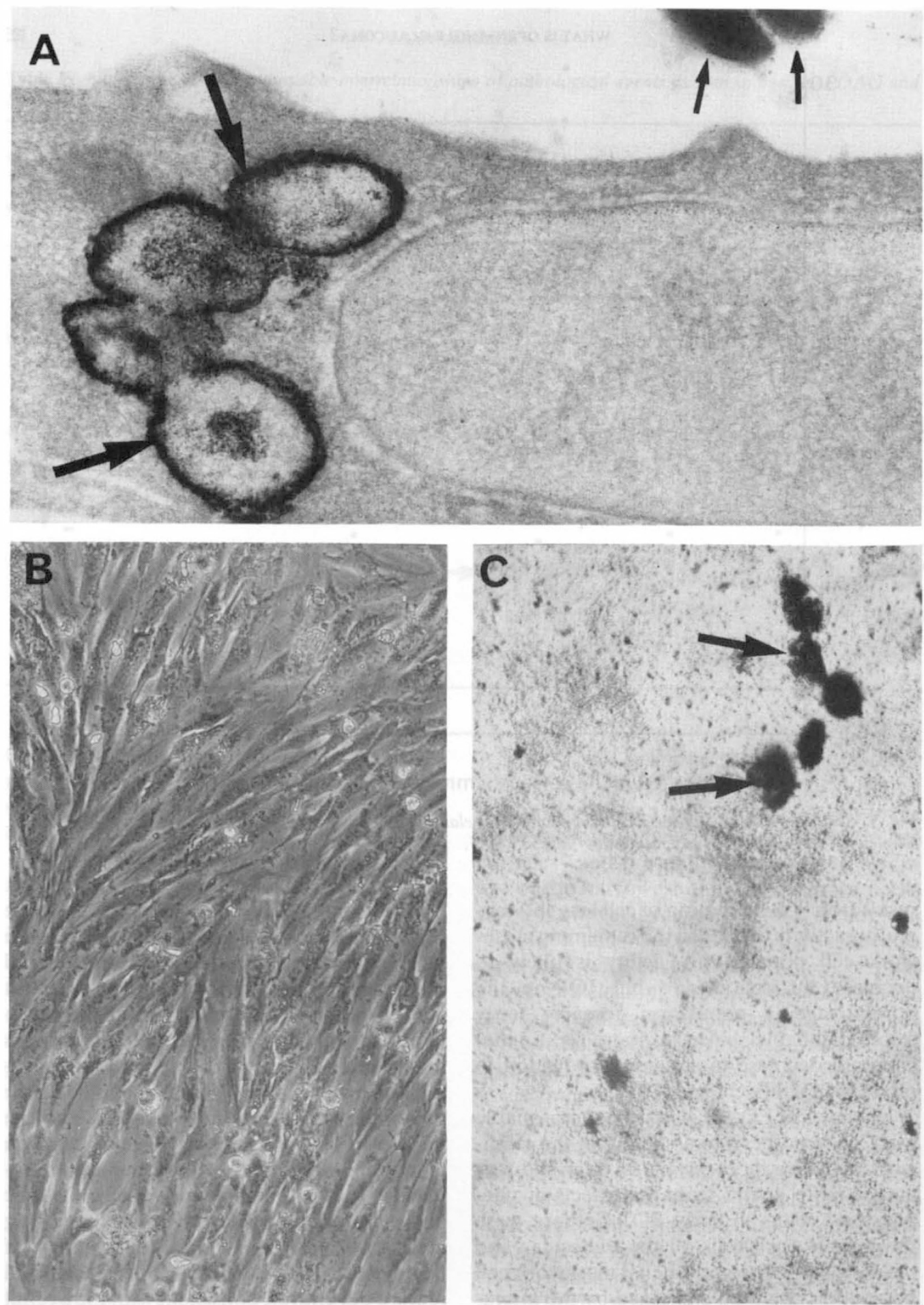

Fig. 8. Phagocytosis by cultured bovine meshwork cells. A) TEM of cells carmine particles are outside the cell (small arrows) and within the cell cytoplasm surrounded by a dark deposit of acid phosphatase reaction product (large arrows). B) Phase micrograph of meshwork cells prior to exposure to IgG coated microspheres $C$ ) Phase micrograph of culture shown in B) After 7 days of daily application of 50,000 microspheres per cell. Only a few cells packed with microspheres remain (arrows). $(A \times 60,000 ; B \times 200 ; C \times 200)$. 

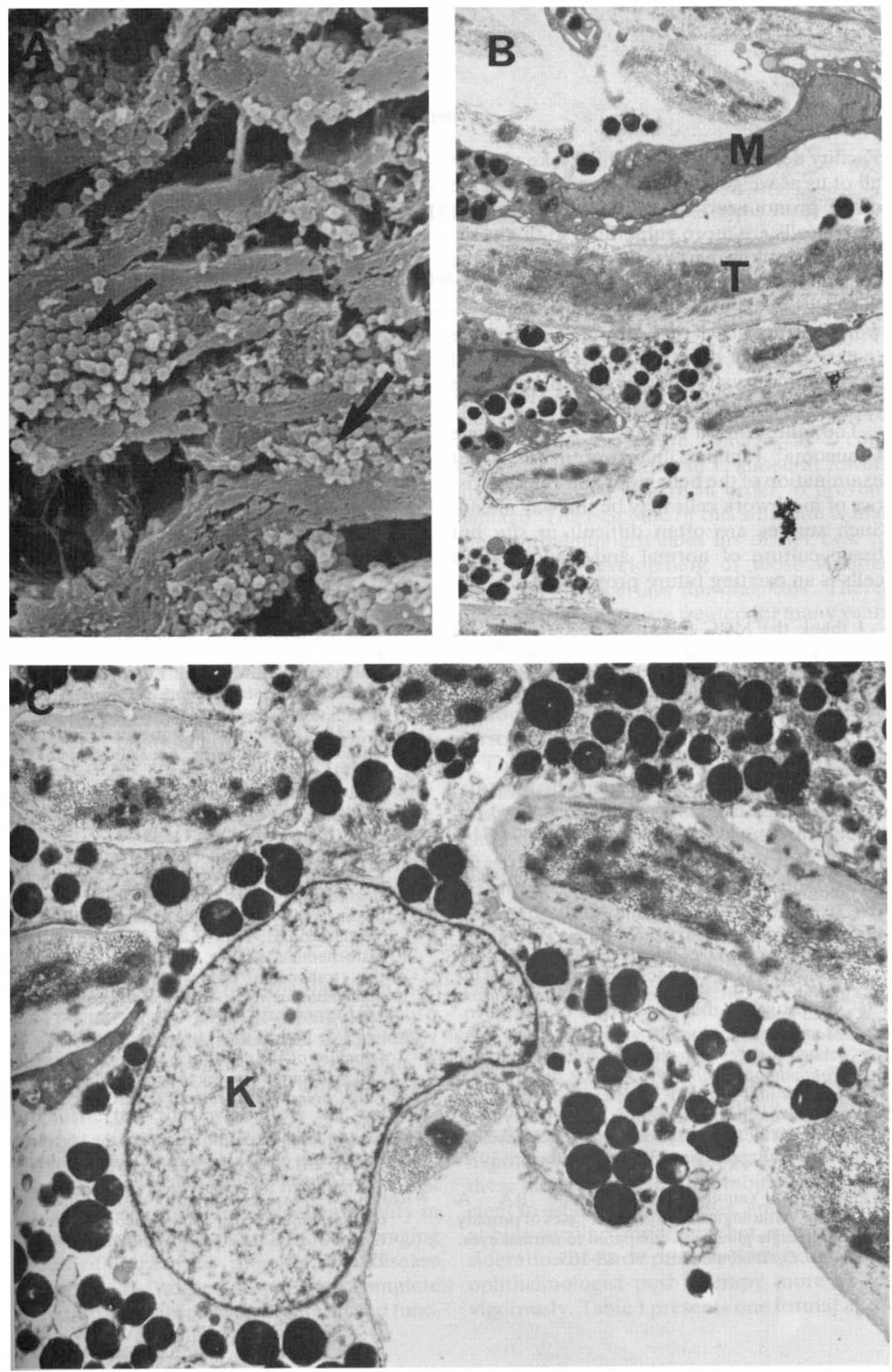

Fig. 9. The meshwork is pigmentary glaucoma. A) SEM showing cells packed with melanin granules (arrows). B) TEM of a migratory meshwork cell $(M)$ leaving a trabecula $(T) . C)$ TEM of lysed meshwork cell with a karyolytic nucleus $(K)$ and cytoplasm full of melanin granules $(A \times 2,000 ; B \times 3,500 ; C \times 8,000)$. 
Facility decreasing events which take place in all of us as we get older (Tables I and IV) are more pronounced in POAGs because the native cells are more vulnerable so that resistance to outflow increases out of step with the age-related decrease in aqueous formation. In SOAGs like pigmentary glaucoma, the meshwork cells are not necessarily more vulnerable than normal but the stimuli which lead to cell death and 'activation' (reparative fibrosis) are more intense.

The question of "What is Open Angle Glaucoma" remains unanswered but closer examination of the behaviour and characteristics of meshwork cells may be the way ahead. Such studies are often difficult in situ but tissue culture of normal and glaucomatous cells is an exciting future prospect.

I thank the MRC (grant no. G8301050N) for their support. I would also like to thank Mr RC Howes, Ms LC Millar, Ms J Day and Ms E Robins for their assistance. Secretarial assistance was provided by Ms P Goodwin.

\section{References}

${ }^{1}$ Alvarado J, Murphy C, Polansky J and Juster R: Age-related changes in trabecular meshwork cellularity. Invest Ophthalmol Vis Sci 1981; 21: 714 27.

${ }^{2}$ Alvarado J, Murphy C and Juster R: Trabecular meshwork cellularity in primary open angle glaucoma and nonglaucomatous normals. Ophthalmolgy 1984; 91: 564-79.

${ }^{3}$ Grierson I, Wang Q, McMenamin PG and Lee WR: The effects of age and antiglaucoma drugs on the meshwork cell population. Res Clin Forums 1982; 4: 69-92.

${ }^{4}$ McMenamin PG, Lee WR and Aitken AN: Agerelated changes in the human outflow apparatus. Ophthalmology 1986; 93: 194-209.

${ }^{5}$ Rohen JW and Lutjen E: Age changes of the trabecular meshwork in human and monkey eyes. In: Brent H, Rohen JW eds. Aging and Development. Stuttgart, New York: Schattauer, 1971, I-36.

${ }^{6}$ Fine BS, Yanoff $M$ and Stone RA: A clinicopathological study of four cases of primary open-angle glaucoma compared to normal eyes. Am J Ophthalmol 1981; 91: 88-105.
${ }^{7}$ Grierson I, Howes RC and Wang Q: Age-related changes in the canal of Schlemm. Exp Eye Res 1984; 39: 505-12.

${ }^{8}$ Grierson I, Robins E, Unger W, Millar L and Ahmed A: The cells of the bovine outflow system in tissue culture. Exp Eye Res 1985; 40: 35-46.

${ }^{9}$ Polansky JR, Wood IS, Maglio MT and Alvarado JA: Trabecular meshwork cell culture in glaucoma research. Ophthalmolgy 1984; 91: 58095.

${ }^{10}$ Lutjen-Drecoll E, Kaufman $\mathrm{P}$ and Barany EH: Light and electron microscopy of the chamber angle structures following surgical disinsertion of the ciliary muscle in the cynomolgus monkey. Invest Ophthalmol Vis Sci 1977; 16: 218-25.

11 Van der Zypen E and Fankhauser F: Ultrastructural changes of the trabecular meshwork of the monkey (Macaca speciosa) following irradiation with argon laser light. Graefes Arch Klin Exp Ophthalmol 1984; 221: 249-61.

12 Rohen JW and Van der Zypen E: The phagocytic activity of the trabecular meshwork endothelium. An electron microscopy study of the vervet (Cercopithecus aethiops). Graefes Arch Klin Exp Ophthalmol 1968, 175: 143-60.

${ }^{13}$ Rohen JW and Lutjen-Drecoll E: Biology of the trabecular meshwork. In: Lutjen-Drecoll $\mathrm{E}$ ed. Basic aspects of glaucoma research. Stuttgart, New York: Schattauer, 1982; 141-66.

${ }^{14}$ Becker B: The decline in aqueous secretion and outflow facility with age. Am J Ophthalmol 1958; 46: 731-6.

15 Segawa K: Electron microscopic changes of the trabecular tissue in primary open-angle glaucoma. Ann Ophthalmol 1979; 11: 49-54.

${ }^{16}$ Rodrigues MM, Katz SI, Fiodart J and Spaeth GL: Collagen, factor VIII antigen and immunoglobulins in the human aqueous drainage channels. Ophthalmology 1980; 87: 337-45.

${ }^{17}$ Lutjen-Drecoll E, Futa R and Rohen JW: Ultrahistochemical studies on tangential sections of the trabecular meshwork in normal and glaucomatous eyes. Invest Ophthalmol Vis Sci 1981; 21: 566-73.

${ }^{18}$ Rohen JW, Futa R and Lutjen-Drecoll E: The fine structure of the cribriform meshwork in normal and glaucomatous eyes as seen in tangential sections. Invest Ophthalmol Vis Sci 1981; 21: 574 85.

${ }^{19}$ Speakman JS and Leeson TS: Site of the obstruction to aqueous outflow in chronic simple glaucoma. Br J Ophthalmol 1962; 46: 321-35.

${ }^{20}$ Rohen JW: Presence of matrix vesicles in the trabecular meshwork of glaucomatous eyes. Graefe's Arch Klin Exp Ophthalmol 1982; 218: 171-6. 